\title{
Thinking, fast and slow
}

In his best-selling book titled Thinking, Fast and Slow, ${ }^{1}$ the Nobel prize laureate Daniel Kahneman explains the 2 systems that drive the way we think and make choices: System One is fast, intuitive, and emotional; System Two is slower, more deliberate, and more logical. Admittedly, there is little in common between the topic of that book and the focus of the current editorial and related original manuscript, which is arrhythmia following the Norwood operation, except for the fact that arrhythmia can be fast and slow. ${ }^{1}$ Nonetheless, we as physicians deal with those 2 thinking systems on a daily basis, and although our decisions are largely based on clinical evidence, some are not, and often are triggered by intuition and anecdotal experiences. Those 2 thinking systems come into play again as we evaluate new information offered by a recent study or research and decide whether or not to adapt any changes in our management strategy.

In the current issue of the Journal, Oster and colleagues ${ }^{2}$ reviewed the data from the Pediatric Heart Network Single Ventricle Reconstruction Trial (SVR Trial) and examined the incidence and risk factors associated with arrhythmias following the Norwood operation and the implication of those postoperative arrhythmias on outcomes. They found that among the 544 patients in their study cohort, 114 $(21 \%)$ experienced at least 1 episode of postoperative tachyarrhythmia, and $21(4 \%)$ experienced at least 1 episode of postoperative atrioventricular block. They also found that although all postoperative arrhythmias were associated with prolonged recovery, only atrioventricular block was associated with diminished transplant-free survival. ${ }^{2}$

Tachyarrhythmia was not infrequent in this study, and was associated with prolonged recovery, although not with diminished survival. ${ }^{2}$ Conclusions from this study, though, are restricted by several limitations. The etiology of tachyarrhythmia is unclear and can be multifactorial, related to metabolic, anatomic, surgical, and demographic factors. ${ }^{3}$ Additionally, various types of arrhythmias can be difficult to distinguish and are often associated with

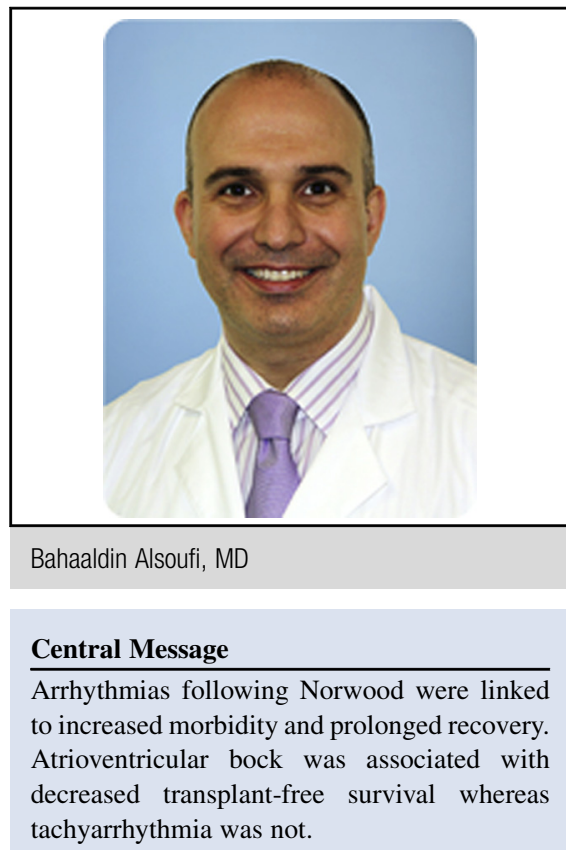

See Article page 638

different provoking factors. Finally, there are great variations in the threshold to treat and the mode of treatment of tachyarrhythmia episodes among institutions and possibly within the same institution. The information provided by the study by Oster and colleagues ${ }^{2}$ suggests that a prophylactic medical treatment in the perioperative period that could successfully decrease the incidence of tachyarrhythmia would be beneficial by decreasing mechanical ventilation and intensive care unit times, with potential subsequent reduction of hospital-related complications and morbidity. In the adult population, prophylactic use of antiarrhythmic agents has been well studied and proved to be successful in decreasing morbidity and resource utilization. ${ }^{4}$ Prophylactic use of antiarrhythmic agents in the pediatric population has been much less studied, and an evidence of the benefit of such a practice does not exist in the heterogeneous pediatric population. The variety in tachyarrhythmia types in those patients and concerns regarding the toxicity of those medications in small infants might have contributed to the existing knowledge gap between the adult and pediatric populations. Although the current study by Oster and colleagues $^{2}$ did not demonstrate an increased risk of interstage mortality in patients who developed tachyarrhythmia following the Norwood procedure, a large number of deaths 
that occur following discharge are of unknown etiology, presumably arrhythmia-related and therefore a prophylactic use of antiarrhythmic agents to decrease that interstage mortality might be beneficial, ${ }^{5}$ although not suggested by Oster and colleagues. ${ }^{2}$ A recent review of the data from the National Pediatric Cardiology Quality Improvement Collaborative (NPC-QIC) demonstrated that not being on digoxin at the time of discharge after the Norwood procedure conferred an increase in the absolute risk of interstage mortality of $11 \%$ and an adjusted odds ratio for interstage mortality of 2.9 , with the assumption that the beneficial mechanism of digoxin is related to both the antiarrhythmic properties and the effects on the neurohormonal axis of heart failure. ${ }^{6}$ Again, challenges in substantiating solid evidence to support such a practice, coupled with concerns about drug toxicity, all limited the interest of programs to adopt those modifications in our outpatient management practice.

Postoperative atrioventricular heart block was less frequent in this study, although it was associated with lower transplant-free survival. ${ }^{2}$ The etiology of heart block in unclear. One potential cause is surgical trauma to the atrioventricular node during atrial septectomy, concomitant procedures such as atrioventricular valve or pulmonary venous connection repair, or postoperative catheter-based intervention. $^{7}$ The findings in the study by Oster and colleagues $^{2}$ support this association, at least in some of those patients. Nonetheless, another etiology that is likely to be connected to postoperative atrioventricular block is myocardial ischemia secondary to poor myocardial protection or ongoing ischemia related to steal or anatomic compromise to coronary perfusion. Despite a comprehensive dataset in the SVR trial, the authors were unable to establish a relationship between atrioventricular block and ischemia. It is plausible that atrioventricular block is essentially a surrogate for the associated conditions, such as concomitant cardiac procedures, poor myocardial protection, or ongoing coronary compromise; all natural risk factors for poor outcome. Therefore, it is not surprising that the placement of a permanent pacemaker did not necessarily improve the outlook of those patients, with only 2 of the 5 patients who received pacemaker successfully proceeding to subsequent palliation stage.,

Moving back to fast and slow thinking, how can we use the current study by Oster and colleagues ${ }^{2}$ to generate an educated, evidence-based conclusion that would improve our inpatient and outpatient management? The answer is difficult, as there are many limitations inherent to the heterogeneous nature of this patient population, the diversity in those fast and slow rhythm disturbances, the management variations between institutions, and the lack of pertinent clinical information despite the comprehensive SVR dataset. It would be very challenging to recreate a well-designed study similar to the SVR trial that was yet unable to solidly examine risk factors and impact of arrhythmias following the Norwood operation. That mission might become possible if the efforts to link multiple registries such as the Society of Thoracic Surgeons, Congenital Heart Surgeons Society, or Pediatric Cardiac Critical Care Consortium were successful and proved to provide valuable additional clinical and follow-up data to allow the formation of this slow, logical decision that is supported by the data. Meanwhile, the fast thinking that is based on intuition and observation might need to take over and, in that case, the study by Oster and colleagues ${ }^{2}$ might incite some centers to adopt a policy to use prophylactic antiarrhythmic agents to decrease the short-term morbidity at least, keeping in mind that atrioventricular block might be itself a marker of poor outcome and potentially not a modifiable one.

\section{References}

1. Kahneman D. Thinking, Fast and Slow. New York. NY: Farrar, Straus and Giroux; 2001.

2. Oster ME, Chen S, Dagincourt N, Bar-Cohen Y, Brothers M, Cain N, et al Development and impact of arrhythmias after the Norwood procedure: A report from the Pediatric Heart Network. J Thorac Cardiovasc Surg. 2017;153: 638-45.e2.

3. Trivedi B, Smith PB, Barker PC, Jaggers J, Lodge AJ, Kanter RJ Arrhythmias in patients with hypoplastic left heart syndrome. Am Heart J. 2011;161:138-44.

4. Raiten JM, Ghadimi K, Augoustides JG, Ramakrishna H, Patel PA, Weiss SJ, et al Atrial fibrillation after cardiac surgery: clinical update on mechanisms and prophylactic strategies. J Cardiothorac Vasc Anesth. 2015;29:806-16.

5. Ghanayem NS, Allen KR, Tabbutt S, Atz AM, Clabby ML, Cooper DS, et al Interstage mortality after the Norwood procedure: results of the multicenter Single Ventricle Reconstruction trial. J Thorac Cardiovasc Surg. 2012;144: 896-906.

6. Brown DW, Mangeot C, Anderson JB, Peterson LE, King EC, Lihn SL, et al Digoxin use is associated with reduced interstage mortality in patients with no history of arrhythmia after stage I Palliation for single ventricle heart disease. J Am Heart Assoc. 2016;5:1-8.

7. Mah DY, Cheng H, Alexander ME, Sleeper L, Newberger JW, Del Nido PJ, et al Heart block following stage 1 palliation of hypoplastic left heart syndrome. J Thorac Cardiovasc Surg. 2016;152:189-94. 